\title{
Die Erfahrung des Heiligen und christliche Publizistik
}

\author{
von Henk Prakke
}

\section{Das Jabr 2000}

Besonders seit der amerikanische Publizist Edward Bellamy im Jahre 1888 seine Utopie "Looking backward $2000-1887$ " veröffentlichte, hat die runde Jahreszahl „2000" in breiten Kreisen faszinierend gewirkt. Das hundertste Jahr des 20. Säkulums sollte das „Paradies auf Erden“ bringen. Wer möchte nicht gerne an eine solche Vorhersage glauben? Doch - je näher wir in der Zeit heranrücken, um so unsicherer werden wir. Das Jahrhundert wird wohl komplettiert werden, aber sicher nicht paradiesisch vollendet. Es sieht manchmal mehr nach Weltuntergang aus. Das war aber schon öfter der Fall. Dennoch ist die Menschheit als solche - sei es mit Schaden und Schanden - immer wieder "noch einmal davongekommen “...

Inzwischen lebt "der Mensch" weiter so wie er ist, mit seinen hellen und seinen dunklen Neigungen. Auch mit seinen Träumereien. Und auch die „Bellamy-Bewegung" wird weiterleben, vielleicht mit Aufschiebung der Zeitgrenze bis auf eine neue runde Zahl: wenn das „Atomzeitalter" es erlaubt, noch so weit zu denken, zur neuen Jahrtausendwende? Der niederländische Publizist Fokke hat schon etwa ein Jahrhundert vor Bellamy über das zukünftige Jahr Dreitausend eine Vorschau veröffentlicht. Aufklärungsgedanken in optima forma: Durch vernünftige Erziehung der Jugend hat man das Verbrechen als solches ausgerottet; es gibt keine Kriminalgesetzgebung mehr, weil diese überflüssig geworden ist; das Land ist "glücklich und gesegnet ${ }^{\text {"1 }}$. Diese Zukunftsphantasie wurde im vorigen Jahre dem lesenden Publikum aufs neue präsentiert in einer Auswahl niederländischer "science fiction“-Erzählun$\operatorname{gen}^{2}$.

\section{Prognostik}

Hellseherisch bin ich nicht veranlagt. Nur einmal in meinem Leben habe ich mich an eine zukunftsbezogene Rede herangewagt. Ich war gebeten worden, zur Jahrhundertfeier meiner ehemaligen Oberrealschule die feierliche Gedenkrede zu halten. Der Altrektor - ein bekannter Historiker - hatte aber in einem zuvor erschienenen (bewundernswerten) Gedenkbuch in Wort und Bild schon alles dargestellt, was erwähnenswert war und - eine Kurzfassung dieser Historie anzubieten - wie auch immer rhetorisch eingebettet - lag mir wirklich nicht. So blieb mir ja nur die Zukunft als Thema übrig. Es kam dann zu einer „futurologischen Causerie"

Nachdem ich zuerst die Chiromantie und die Astrologie kurz gestreift hatte, kam ich schon bald zu der Feststellung, daß nur empirisch festgestellte Fakten, exakte Statistiken und sorgfältig berechnete Trends zur gediegenen Schlußfolgerung eines zu erwartenden Hergangs führen können. Nur in dieser Weise kommen wir zu sicheren Prognosen, zu einer zuverlässigen Futurologie. Soweit gekommen, fiel ich mir selbst

Dr. Henk Prakke, Meppen-Zweeloo (Dr.), Niederlande, ist emeritierter ordentlicher Professor für Publizistik an der westfälischen Wilhelms-Universität in Münster. 
aber schon ins Wort. Naturwissenschaftlich möge man z. B. mit Raumfahrtprogrammen unglaublich haarscharf Vorhersagen machen können, geisteswissenschaftlich entdeckt man aber schon bald, daß „es überall menschelt". Gibt es nicht schon nebeneinander eine "Establishment Futurologie" und eine „Kritische Futurologie“? Und nicht zu verneinen ist, daß aufgrund derselben faktischen Gegenwart der eine mit Edward Bellamy oder mit Karl Marx zu einem utopischen Jahr 2000 blickt, der andere aber mit George Orwell oder Aldous Huxley zu einer negativen „1984“Utopie.

\section{Kommunikation im Jabre 2000}

Ginge man davon aus, daß das „Nirgendwo" der Utopie sich im Jahre 2000 nirgendwo - so oder so - verwirklichen ließe, so könnte zur „utopischen Kommunika-

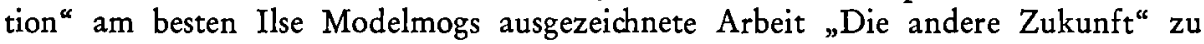
Rate gezogen werden'.

Ist man der Meinung, daß ein "diesseitiges“, von Menschen geschaffenes „Paradies“ nicht zu erwarten ist, dann bleibt man für eine mögliche Vorschau („möglich“, weil das Unerwartete von Erfindungen und ... Kalamitäten immer mit im Spiel ist!) angewiesen auf den schon angedeuteten Weg von Fakten, Statistiken und Trends - womit ein voll besetztes Publizistikseminar allein ein ganzes Semester wissenschaftlich beschäftigt sein könnte. Als Seminarleiter würde ich am Anfang hypothetisch feststellen, daß

a) materiell: die technischen Kommunikationsmöglichkeiten sich immer weiter und schneller entwickeln werden (Fragen: wie, wo, wann?);

b) spirituell: eine noch weitergreifende Kommunikationsexplosion, das "gesellschaftliche Zwiegespräch" immer mehr zu einer neuen babylonischen Sprachenverwirrung führen würde (Fragen des kommunikativen Verhaltens und der Wirkung) ${ }^{5}$.

Als Wissenschaftler möchten wir selbstverständlich diese Vorschau so objektiv wie möglich darstellen. In der Praxis haben wir jedoch damit zu rechnen, daß mancher weltanschaulich Engagierte die Vorschau zur programmäßigen Planung umdenkt. Dieses Engagement kann - wenn einflußreich unterstützt - zur "sich-selbsterfüllenden Prophetie" führen.

\section{Beitrag der Kirche?}

Sobald die Frage nach dem "Beitrag der Kirche“ gestellt wird, ist man meistens schon beim programmierenden Engagement gelandet.

Versucht man den "Trend“ des 20. Jahrhunderts zu dieser Frage zu erkunden, dann zeigen die abendländischen Statistiken eine fortwährende Dekonfessionalisierung und Säkularisierung. Viele Aufgaben, die einst typisch kirchliches Arbeitsgebiet waren (Diakonalsorge, Krankenpflege, Unterrichtswesen), sind allmählich von der weltlichen Obrigkeit übernommen und damit professionalisiert. Tendenz: Der Berufene ist dem Berufler gewichen.

Markant ist die Klage zweier Krankenschwestern aus Ghana, die als „Entwicklungshilfe ${ }^{"}$ ein Jahr in Europa arbeiten durften: Wir haben vieles zugelernt, sind aber recht dankbar, wieder heimkehren zu können: Es ist hier alles abscheulich materialistisch eingestellt und - Gott haben wir in den Gesprächen hier sehr 
vermißt. Werden die "jungen“ Kirchen von dort im Jahre 2000 ihre Missionare ins alte Abendland schicken müssen?

Ob dieser Säkularisierungstrend des Westens sich weiter fortsetzen wird, oder ob die Kirche (in der Gespaltenheit ihrer Erscheinungsformen: die Kirchen) im letzten Viertel des Jahrhunderts noch an Einfluiß zurückgewinnen wird? Es sind Gegenkräfte tätig. Erst spät hat man im allgemeinen die Bedeutung der neuen „Massenmedien" für die kirchliche Botschaft entdeckt. Für die "propaganda fidei“ hat man die erfolgreiche kommerzielle Werbung zum Vorbild erhoben. Die Durchführung der altehrwürdigen Gebote und Verbote hat man, dem Zug der Zeit sich fügend, allmählich abgeschwächt. $\mathrm{Ob}$ es reicht für eine Wiederentdeckung des Christentums als Kern der abendländischen Kultur, einer Kultur, die in der erreichten Wohlstandsgesellschaft einen bedenklichen Niedergang erleidet? Grundsätzlich leider nicht. Die „imago“ der Kirche als solcher hat bei dem Säkularisierungsprozeß eben zu stark gelitten. Die Demokratisierung des Luxus war offensichtlich einfacher zu erreichen als die der Kultur.

\section{Das Doppelgebot von Matthäus XXII.}

Klingt dies alles zu pessimistisch? Ich weiß: Man darf nicht ohne Grund verallgemeinern. Und ich weiß auch, daß es noch immer blühende Kirchengemeinden und noch immer auch begnadete "Diener des göttlichen Wortes" gibt.

Um die Jahrhundertwende geboren, habe ich dieses ganze Säkulum, von der Bell' Epoque meiner Kindheit an, miterlebt - mit seinem $\mathrm{Auf}$ und $\mathrm{Ab}$ von Kriegen und Krisen, vom Segnen der Waffen 1914 bis zum kirchlichen Verständnishaben für Terroristen als Freiheitskämpfer im Jahre 1977. Und was darf man dann dazu noch im „Kronjahr" 2000 erwarten? Die Dissonanz ist zu sehr vorherrschend, nicht nur in der jeweiligen musischen Publizistik, sondern auch im jeweiligen Lebenskode, wo einmal doch die Harmonie (wie schlecht auch manchmal praktiziert) Modell stand.

Dennoch: Die Einzigartigkeit des Christentums zeigt sich noch immer klar, wenn man z. B. als Publizistikforscher einen wissenschaftlichen Bilderatlas von Gottesdarstellungen in aller Welt sich ansieht ${ }^{7}$. Nirgendwo "publiziert sich" eine göttliche Menschennähe und Menschenliebe so wie in den Bildern der "Passion Christi“.

Es ist eben diese Menschennähe, die für manchen Prediger und für manchen Kirchgänger den Glauben vereinfacht hat zu einer „sozialen Religion“. Man befindet sich auf einmal außerhalb der Kirche in einer Gesellschaft von vielen, die (der Kirche möglicherweise schon in einer früheren Generation - entfremdet) sich wohl einsetzen wollen für mancherlei Sozialarbeit, unbewußt sich damit aber einer christlichen Erblassung widmen.

Diese Vereinfachung ist jedoch eine Einseitigkeit, die sich rächt und die im Wider-

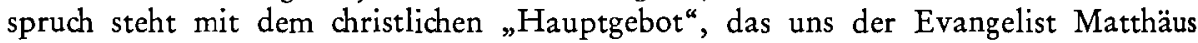
überliefert ${ }^{8}$ : Das vornehmste Gebot - Liebe zu Gott; das andere, dem ersten gleich - Liebe zum Nächsten. Dieses Doppelgebot - als schwerste Aufgabe dem Menschen je gestellt - gibt durch seine vertikale Gerichtetheit der horizontalen „Sozialreligion" erst ihren wesentlichen Sinn und ihre wahre Gesinnung.

Es gibt keine Statistik, wie viele religiös-veranlagte Menschen eben durch diese horizontale Einseitigkeit und die damit manchmal verknüpfte "entseelende“ Professionalisierung der Liebesarbeit (gewerkschaftlich mammonisiert) kirchlich obdach- 
los geworden sind; - keine Statistik, wie viele kirchlich enttäuschte Menschen zum seelischen "Selbstversorger" geworden sind in der "Kirche von ungekerbtem Holz", wo Vögel den Chorgesang betreuen und der Wind in den Tannen das nicht mit Computern zu fassende Mysterium unseres Daseins verkündet und damit zur "Stimme Gottes" wird: eine Kommunikation ganz eigener Art. Die Einsamkeit in der modernen Masse ist manchmal größer als die in der Ein-samkeit. Zwei Gruppen von Unkirchlichen - Produkte des 20. Jahrhunderts, für welche die übliche kirchliche Publizistik generell keine Lösung - und sicher keine Erlösung - bietet. Ist dies Schwarzseherei - oder programmierendes Engagement?

\section{Religionspublizistik}

Ein Zitat eigener Mache zum Schluß: „Der politischen Publizistik ist wissenschaftlich und praktisch die größte Aufmerksamkeit gewidmet worden. Die kirchliche Publizistik hat man im allgemeinen abgespeist mit der empfohlenen und zögernd praktizierten Nachahmung, weltlicher Publizistik؛. Daß die kirchliche Publizistik grundsätzlich als eine publizistische Kategorie sui generis anzusehen ist, wurde auch in kirchlichen Krisen - nicht klar genug gesehen.

In der Religionssoziologie geht P. J. Bouman davon aus, daß zu allen Zeiten und bei allen uns bekannten Völkern die Trennungslinie zwischen dem Profanen und dem Heiligen (oder Magischen) in der menschlichen Gesellschaft eine Rolle gespielt hat ${ }^{9}$. Diese ,Erfahrung des Heiligen' nun muß wohl Kernpunkt kirchlicher Kommunikation sein, und die eigene Kategorie, die wir andeuteten, könnte als Wissenschaftsbereich (parallel zur Religionssoziologie) als Religionspublizistik bezeichnet werden. Die kirchliche Publizistik wäre Unterteil dieses ganzen Bereiches. Als Wissenschaft wäre dieser Bereich der Religionspublizistik zu definieren als die Lehre der Erfahrung des Heiligen in ihrer Wirkung auf das gesellschaftliche Zwiegespräch. ${ }^{* 10}$

In diesem Sinne könnte die Kirche einen sehr wesentlichen Beitrag liefern zu einer entsäkularisierenden Kommunikation im Jahre 2000 (3000?).

\section{Anmerkungen:}

1. Arend Simonsz Fokke: Het toekomende jaar 3000, eene mijmering. Amsterdam 1793. Lebensbericht Fokke (Amsterdam 1755-1812). In: Nieuw Nederlandsch Biografisch Woordenboek. Leiden 1937, X, 264-265. Über die Zukunft als Steigerung der Gegenwart vgl. auch: Henk Prakke: Vom Sinn der Publizistik. Assen 1965, 11 ff.

2. Manuel van Loggem (Hrsg.): Het toekomende jaar 3000, science-fictionverhalen van literaire schrijvers. Antwerpen 1976, $179 \mathrm{ff}$.

3. H. J. Prakke: Garven. Assen 1975, 80 ff.: Sdhoolgaan in het hart van de Gouden Delta, een futurologische causerie.

4. Ilse Modelmog: Die andere Zukunft. Zur Publizistik und Soziologie der utopischen Kommunikation. Düsseldorf 1970.

5. Henk Prakke, mit Franz W. Dröge, Winfried B. Lerg und Michael Schmolke: Kommunikation der Gesellschaft. Einführung in die funktionale Publizistik. Münster 1968.

6. Conny Huysmans: Ghanese verpleegsters snakken naar huis. In: „De Telegraaf“, 21. April 1977.

7. F. Sierksma: De mens en zijn Goden. Amsterdam 1959.

8. Matth. 22,36-40: „Meister, welches ist das vornehmste Gebot im Gesetz? Jesus aber sprach zu ihm: „Du sollst lieben Gott, deinen Herrn, von ganzem Herzen, von ganzer Seele und von ganzem Gemüte. Dies ist das vornehmste und größte Gebot. Das andre 
aber ist dem gleich: ,Du sollst deinen Nächsten lieben wie dich selbst'. An diesen zwei Geboten hängt das Gesetz und die Propheten."

9. P. J. Bouman: Einführung in die Soziologie. Stuttgart ${ }^{2} 1960,129$.

10. Henk Prakke: Vorwort zu Franz-Josef Eilers SVD: Christliche Publizistik in Afrika, Eine erste Erkundung. St. Augustin 1964.

\section{S U M M A R Y}

Although the year 2000 may not bring about the paradise anticipated by some Utopians, it might be possible in science to make a few predictions about the future. In the arts, however, human limitations will always be obvious. The futurology of communications could itself provide a full year's work for a seminar dealing with the new technical possibilities for communications, and the even more important consequences of the communications revolution for the "Social Dialogue". The trend towards the secularisation of the Churches in the West cannot be denied. The importance of mass media in spreading the Christian message came as a very late discovery. Over-simplification of Christian beliefs in an effort towards greater "humanisation" led to a horizontal "social religion" which seems to lack the vertical line of the love of God as expressed in St. Matthew, h. 22. The experience of the "holy" ("das Heilige") should be at the centre of Christian communications, which, as religious communications, can be defined as the science of the experience of the "holy" and its impact on social dialogue.

\section{RESUME}

Même l'an 2000 n'apportera pas ce paradis que certains utopistes ont attendu. Même sí l'on peut faire scientifiquement des prévisions, à l'aide de recherches exactes, on découvre cependant bientôt de façon intellectuelle que partout rien est parfait. Une communication futuriste pourrait occuper pendant tout un semestre un séminaire pour publicistique bien plein, et ce avec les possibilités de communication techniques en voie de développenent, et l'explosion de communication qui touche encore plus loin avec ses conséquences sur le „dialogue social“. Chez les Eglises d'Orient, il ne faut pas nier la tendance à la sécularisation. Ce n'est qu'assez tard qu'on a découvert la signification des mass media sur le message ecclésiastique. La simplification de la foi chrétienne, afin de la mettre plus à la portée de l'homme, a mené à une "religion sociale» horizontale qui semble manquer à la verticale de l'amour divin du premier commandement chez Matthieu 22. L'expérience de ce qui est saint devrait redevenir le noyau de la publicistique chrétienne qui, en tant que publicistique de religion, peut être définie comme »l'enseignement de l'expérience de ce qui est saint dans ses effets sur le dialogue social «.

\section{RES UMEN}

Tampoco el año 2000 traerá consigo el paraíso que muchos utopistas esperan. A pesar de que, gracias a investigaciones exactas, pueden hacerse pronósticos científicos, en las ciencias del espíritu se descubren "lagunas" por doquier. La comunicación futurista podría dar tema durante un curso a un seminario muy concurrido que estudiara las posibilidades de la communicación, la explosión numérica de los medios y sus consecuencias para el ndiálogo social«. En la Iglesia de occidente no puede ocultarse un proceso de secularización. Se descubrió demasiado tarde la significación de los medios de difusión para el mensaje evangélico. La simplificación de la fe cristiana a nivel humano condujo a una mreligión social“ horizontal, que adolece de la verticalidad del amor divino expresada en Marcos 22. La experiencia del santo debería ser de nuevo punto central del publicismo cristiano, que como publicismo podría definirse: "La doctrina de la experiencia del santo proyectada al diálogo social «. 